\title{
MODELING HETEROGENEOUS POPULATIONS OF THERMOSTATICALLY CONTROLLED LOADS USING DIFFUSION-ADVECTION PDES
}

\author{
Scott Moura* \\ Victor Ruiz \\ Dept. of Mechanical and Aerospace Engineering \\ University of California, San Diego \\ La Jolla, California 92093-0411 \\ Email: smoura@ucsd.edu,viruiz@ucsd.edu
}

\author{
Jan Bendtsen \\ Dept. of Electronic Systems \\ Aalborg University \\ 9210 Aalborg \\ Denmark \\ Email: dimon@es.aau.dk
}

\begin{abstract}
This paper focuses on developing a partial differential equation (PDE)-based model and parameter identification scheme for heterogeneous populations of thermostatically controlled loads (TCLs). First, a coupled two-state hyperbolic PDE model for homogenous TCL populations is derived. This model is extended to heterogeneous populations by including a diffusive term, which provides an elegant PDE control-oriented model. Second, a novel parameter identification scheme is derived for the PDE model structure, which utilizes only boundary measurements and aggregated power measurements. Simulation results against a Monte Carlo model of a large TCL population demonstrate the usefulness of the approach. The proposed model and parameter identification scheme provide system critical information for advanced demand side management control systems.
\end{abstract}

\section{INTRODUCTION}

One of the main challenges in achieving significant penetration of renewables in modern energy supply systems is their inherent variability. To this end, demand side management has been gaining attention in recent years as a means of achieving better balance between supply and demand in modern power grids, in the presence of intermittent power sources [1-3].In particular, control of aggregated thermostatically controlled loads (TCLs) provides a promising opportunity to mitigate the mismatch between power generation and demand, thus enhancing grid reliability and enabling renewable energy penetration [4-6]. However, to exploit the full potential of demand side management, one requires mathematical models that describe the dy-

\footnotetext{
*Address all correspondence to this author.
}

namical behavior of the TCL population as accurately as possible [5, 7-11].

Indeed, to manipulate the individual units' power consumption, an entity interested in using TCLs for power demand/supply balancing-e.g., a utility company-in principle requires measurements of all states in the system. Moreover, they require a means of either forcing or coercing the consumers to consume more or less power. Thus, controlling individual units typically requires detailed local measurement and feedback of current energy and power demands [12]. Implementing such infrastructure has been shown to be feasible in practice for limited numbers of units [13].

For large portfolios of consumers, however, controlling individual units directly is bound to lead to a heavy communication and computational burden on the system. Rather than attempting to control every consumer individually, methods for modeling, estimating and controlling the behavior of large populations of consumers have come into focus recently [4-6]. Under this paradigm one manipulates the operating conditions of the entire population to shape total power demand, while avoiding user discomfort.

In this paper, we examine modeling and real-time identification of the distribution of temperature states in aggregated TCL populations. This information can be utilized to accurately manipulate the temperature set points to shape the aggregated TCL power consumption. Given accurate and timely information about how the power consumption of a population of TCLs can be adjusted, it becomes possible to use the population as a fast "virtual power plant" that can provide auxiliary services to the grid. This includes provision of frequency control reserves and peak shaving (in reaction to unforeseen disturbances) to a 
more market-based mode of operation, where portions of energy (in the form of shifted consumption) can be traded on electricity spot markets. Obviously, more precise information about the storage reserves available provides the operator with greater flexibility in terms of providing such services in a reliable and timely manner without causing discomfort to the consumers.

To this end, we consider a large population of TCLs and derive a partial differential equation-based model of the temperature evolution. This model does not assume a constant "duty cycle" of the loads. More precisely, we present two PDE models of TCL populations. The first model assumes all TCLs are identical. The second model includes a diffusion term to account for the actual heterogeneity amongst the TCL parameters (e.g., thermal time constants, temperature dead-band limits). In doing so, we expand upon existing PDE-based TCL models which either do not take heterogeneity explicitly into account [10], or model heterogeneity using Markov Chains [14,15]. We also present a novel scheme for identifying the parameters in the PDE model based on measurements of aggregated power consumption and the rate at which loads switch on/off.

The outline of the paper is as follows. We start in Section 2 by deriving a model for populations of identical TCLs. Then we extend this model to heterogeneous populations by including a diffusive term. Section 3 presents a novel identification scheme for the PDE models. Section 4 presents simulation studies of the identification scheme, whereupon Section 5 summarizes the main contributions of this work.

\section{MODELS OF POPULATIONS OF TCLS}

We first consider the following lumped thermal mass, hybrid ODE model of a single TCL. For the $i$ 'th consumer let the controlled thermal zone and ambient temperature (living spaces, refrigerators, cold storages etc.) be denoted by $T_{i}$ and $T_{\infty, i}$, respectively. Assume the hardware is purely on/off-regulated. The temperature dynamics are governed by

$$
\begin{aligned}
& \dot{T}_{i}(t)=\frac{1}{R_{i} C_{i}}\left(T_{\infty, i}-T_{i}(t)-s_{i}(t) R_{i} P_{i}\right), \quad i=1,2, \ldots, N \\
& s_{i}(t)=\left\{\begin{array}{lll}
0 & \text { if } s_{i}(t-\varepsilon)=1, & T_{i}(t) \leq T_{\min , i} \\
1 & \text { if } s_{i}(t-\varepsilon)=0, & T_{i}(t) \geq T_{\max , i} \\
s_{i}\left(t^{-}\right) & \text {otherwise }
\end{array}\right.
\end{aligned}
$$

where $C_{i} \in \mathbb{R}^{+}$is the thermal capacitance $\left(\mathrm{kWh} /{ }^{\circ} \mathrm{C}\right), R_{i} \in \mathbb{R}^{+}$is the thermal resistance $\left({ }^{\circ} \mathrm{C} / \mathrm{kW}\right)$ and $P_{i} \in \mathbb{R}$ is the (constant) heating/cooling power supplied by the hardware when switched on. Thus, $1 / R_{i} C_{i}$ is the thermal time constant of the $i$ 'th TCL. Variable $s_{i} \in\{0,1\}$ is binary-valued and determines whether or not the hardware is turned on. In practice, it switches status whenever the internal temperature encounters the limits of a pre-set temperature span $\left[T_{\min , i}, T_{\max , i}\right] \subset \mathbb{R}$.

Limits $T_{\min , i}$ and $T_{\max , i}$ are related to the $i$ 'th TCL's setpoint
$T_{\mathrm{sp}, i}$ through the fixed relations

$$
T_{\min , i}=T_{\mathrm{sp}, i}-\frac{\Theta_{i}}{2}, \quad T_{\max , i}=T_{\mathrm{sp}, i}+\frac{\Theta_{i}}{2}
$$

where $\Theta_{i}$ is the width of the temperature interval. Furthermore, the cumulative power consumption of the TCL population at any given time $t$ can be computed as

$$
P(t)=\sum_{i=1}^{N} \frac{P_{i} s_{i}(t)}{\eta_{i}}
$$

where $\eta_{i}$ is the coefficient of performance for the $i$ 'th heating/cooling unit. Assuming that it is possible to adjust the setpoint $T_{\mathrm{sp}, i}$ for a large number of consumers, it becomes possible to shape the aggregated power consumption. Thus, $T_{\mathrm{sp}, i}$ is of interest for control purposes. In this paper we focus on modeling and identification, however, and will not include the setpoint explicitly in the following models.

\subsection{Homogeneous population}

Consider a large population of $N$ TCLs, satisfying the dynamics described above. Let the continuously differentiable functions $u(T, t)$ and $v(T, t)$, both defined on the spaces $\left[T_{\min }, T_{\max }\right] \times \mathbb{R}^{+} \rightarrow \mathbb{R}$, denote the distributions of loads (relative to $N$ ) at temperature $T$ and time $t$ in the on and off states, respectively. By considering the 'flow' of TCLs along the temperature axis in either the positive or negative directions, and taking limits, it is possible to derive the following PDE model of homogeneous populations of TCLs.

For modeling purposes, we initially assume that all parameters, including $T_{\min , i}$ and $T_{\max , i}$, are equal for all TCLs; i.e., all the TCLs are assumed to be identical. Let $\bar{R}, \bar{P}$ and $\bar{C}$ denote the population-wide values of $R_{i}, P_{i}$ and $C_{i}$, respectively. Consider a specific temperature $T \in\left[T_{\min , i}, T_{\max , i}\right]$. The "flux" of TCLs traversing this temperature at time $t$ in the increasing and decreasing direction can be written as

$$
\phi(T, t)=\left.u(T, t) \frac{d T}{d t}\right|_{s=1} \text { and } \psi(T, t)=\left.v(T, t) \frac{d T}{d t}\right|_{s=0}
$$

respectively. Using (1) we get

$$
\begin{aligned}
& \phi(T, t)=\frac{1}{\bar{R} \bar{C}}\left(T_{\infty}-T(t)-\bar{R} \bar{P}\right) u(T, t) \\
& \psi(T, t)=\frac{1}{\bar{R} \bar{C}}\left(T_{\infty}-T(t)\right) v(T, t)
\end{aligned}
$$

Consider the small control volume of width $\delta T$ shown in Fig. 1. 


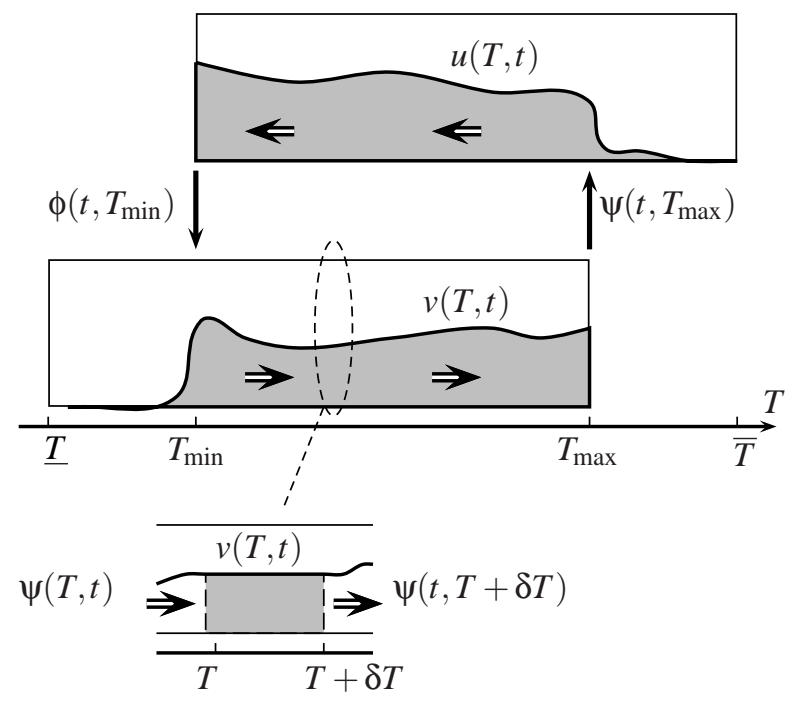

Figure 1. Illustration of transport PDE representation of temperature distributions; $u(T, t)$ is the on state distribution, while $v(T, t)$ is the off state distribution. The bottom part of the figure shows a zoom on a small control volume of width $\delta T$, in which $v(T, t)$ is assumed to be constant.

Using limit arguments, we get the relation

$$
\begin{aligned}
\frac{\partial v}{\partial t}(T, t) & =\lim _{\delta T \rightarrow 0}\left[\frac{\psi(T+\delta T, t)-\psi(T, t)}{\delta T}\right] \\
& =\frac{\partial \psi}{\partial T}(T, t) \\
& =-\frac{1}{\bar{R} \bar{C}}\left[T_{\infty}-T(t)\right] \frac{\partial v}{\partial T}(T, t)+\frac{1}{\bar{R} \bar{C}} v(T, t)
\end{aligned}
$$

for the TCLs in the off state and, correspondingly,

$$
\begin{aligned}
\frac{\partial u}{\partial t}(T, t) & =-\frac{\partial}{\partial T}\left[\frac{1}{\bar{R} \bar{C}}\left(T_{\infty}-T(t)-\bar{R} \bar{P}\right) u(T, t)\right] \\
& =-\frac{1}{\bar{R} \bar{C}}\left[T_{\infty}-T(t)-\bar{R} \bar{P}\right] \frac{\partial u}{\partial T}(T, t)+\frac{1}{\bar{R} \bar{C}} u(T, t)
\end{aligned}
$$

for the TCLs in the on state.

As the temperatures of TCLs in the on state reach $T_{\min }$, they switch to the off state, and vice versa at $T_{\max }$. After some finite time, no TCLs will exist outside the interval $\left[T_{\min }, T_{\max }\right]$, in which case the couplings indicated in Fig. 1 can be stated as

$$
\begin{aligned}
\phi\left(t, T_{\max }^{-}\right)+\psi\left(t, T_{\max }\right) & =0 \\
\psi\left(t, T_{\min }^{+}\right)+\phi\left(t, T_{\min }\right) & =0
\end{aligned}
$$

or

$$
\begin{aligned}
& u\left(t, T_{\max }\right)=-\frac{T_{\infty}-T_{\max }}{T_{\infty}-T_{\max }-\bar{R} \bar{P}} v\left(t, T_{\max }\right) \\
& v\left(t, T_{\min }\right)=-\frac{T_{\infty}-T_{\min }-\bar{R} \bar{P}}{T_{\infty}-T_{\min }} u\left(t, T_{\min }\right)
\end{aligned}
$$

To summarize, the homogeneous TCL population model is:

$$
\begin{aligned}
u_{t}(T, t) & =\alpha \lambda(T) u_{T}(T, t)+\alpha u(T, t) \\
v_{t}(T, t) & =-\alpha \mu(T) v_{T}(T, t)+\alpha v(T, t) \\
u\left(T_{\max }, t\right) & =q_{1} v\left(T_{\max }, t\right) \\
v\left(T_{\min }, t\right) & =q_{2} u\left(T_{\min }, t\right)
\end{aligned}
$$

where subscripts $(\cdot)_{T}$ and $(\cdot)_{t}$ denote partial derivatives of $(\cdot)$ with respect to temperature and time, respectively. The parameters $\alpha, \lambda(T), \mu(T), q_{1}, q_{2}$ are given by

$$
\begin{aligned}
\alpha & =\frac{1}{\bar{R} \bar{C}}>0 \\
\lambda(T) & =-\left(T_{\infty}-T-\bar{R} \bar{P}\right)>0 \\
\mu(T) & =T_{\infty}-T>0 \\
q_{1} & =-\frac{T_{\infty}-T_{\max }}{T_{\infty}-T_{\max }-\bar{R} \bar{P}} \\
q_{2} & =-\frac{T_{\infty}-T_{\min }-\bar{R} \bar{P}}{T_{\infty}-T_{\min }}
\end{aligned}
$$

Note that, unlike [10], we have retained the temperature variation around the setpoint, which ultimately produces the reaction terms in (10)-(11).

The power consumption of the total population may be obtained by integrating the distribution of TCLs in the on-state:

$$
P(t)=\frac{\bar{P}}{\eta} \int_{T_{\min }}^{T_{\max }} u(T, t) d T
$$

where $\bar{P}$ is the (constant) power delivered to each TCL in the on state and $\eta$ is the coefficient of performance for the TCLs.

Figure 2 demonstrates the aggregated behavior for 50 identical TCLs. The left plots show how the TCLs alternate between the on and off states while remaining within the operation band. The TCLs were initiated at random temperatures, normally distributed around $20^{\circ} \mathrm{C}$, all in the off state. As can be seen, the power drawn by the population oscillates with a constant amplitude, since the TCLs have identical duty cycles. We say that the TCLs are synchronized.

The right plots in Fig. 2 show a similar situation, however now the individual TCL time constants are drawn from a random distribution, thus making the population heterogenous. The 

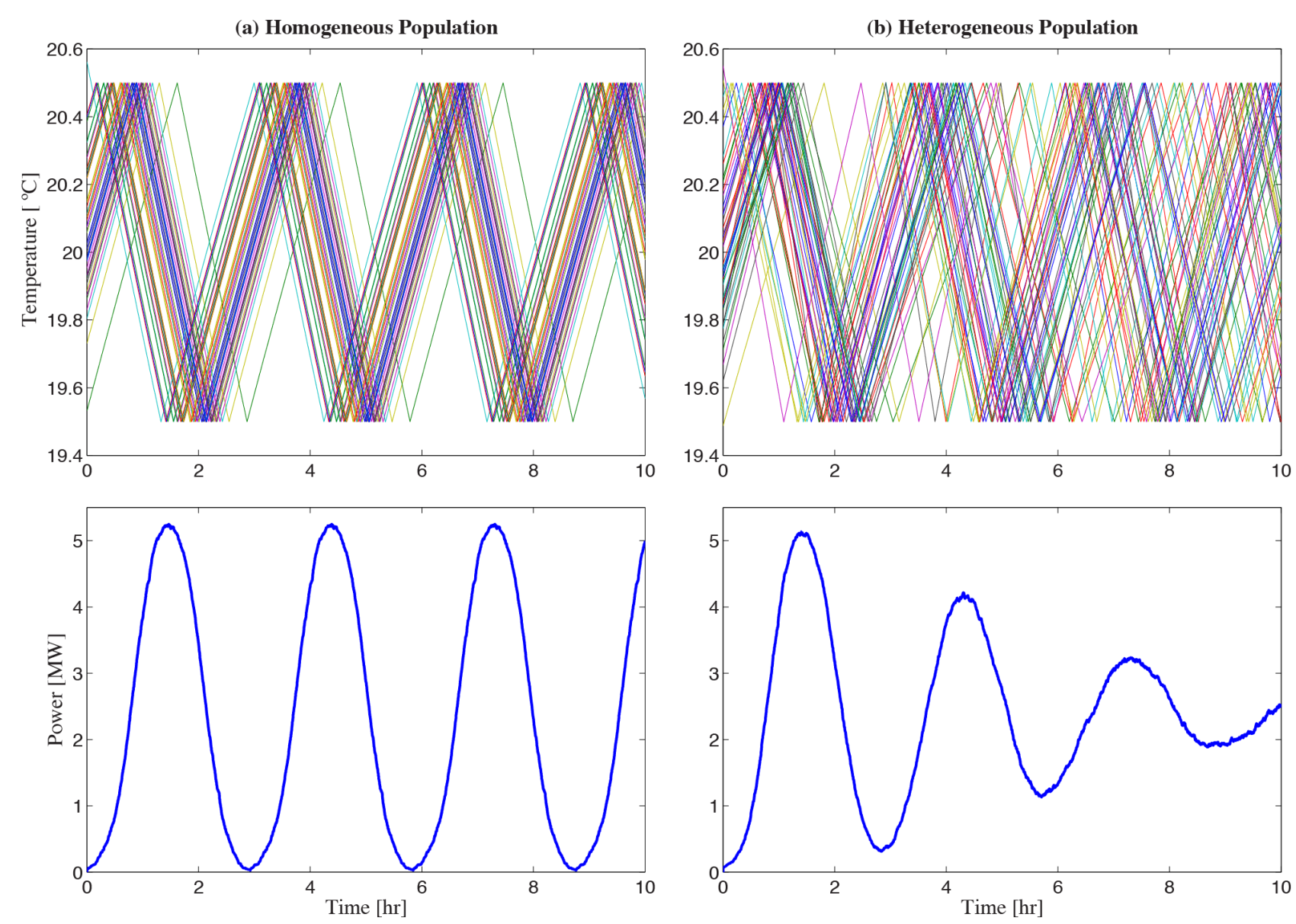

Figure 2. Evolution of temperature for 50 TCLs from (a) homogeneous and (b) heterogeneous populations. In each case, the population was initialized with all TCLs in the off state, with temperature distributed according to $u_{0}(T), v_{0}(T)$ in Table 1. Note the TCLs remain in synchrony for the homogenous population. In contrast, the temperature distribution diffuses in the heterogeneous case. This observation motivates the heterogeneous model in Section 2.2.

corresponding total power consumption exhibits damped oscillations, due to different time constants that cause the individual TCLs to gradually de-synchronize. Similar behaviors occur if other parameters such as $\Theta_{i}$ or $T_{\infty, i}$ are allowed to vary across the TCL population.

\subsection{Heterogeneous population}

Motivated by the damping phenomenon (so-called desynchronization) observed in the Monte Carlo simulations of heterogeneous populations, we propose to add a diffusion term to the advection model (10)-(11):

$$
\begin{aligned}
u_{t}(T, t) & =\alpha \lambda(T) u_{T}(T, t)+\alpha u(T, t)+\beta u_{T T}(T, t) \\
v_{t}(T, t) & =-\alpha \mu(T) v_{T}(T, t)+\alpha v(T, t)+\beta v_{T T}(T, t) \\
u\left(T_{\max }, t\right) & =q_{1} v\left(T_{\max }, t\right) \\
v\left(T_{\min }, t\right) & =q_{2} u\left(T_{\min }, t\right)
\end{aligned}
$$

In order to preserve well-posedness of the PDE system, two more boundary conditions are required. These extra conditions are selected to reflect the fact that the number of TCLs, $N$, which can be expressed in terms of $u$ and $v$ by

$$
N(t)=\int_{T_{\min }}^{T_{\max }} u(T, t) d T+\int_{T_{\min }}^{T_{\max }} v(T, t) d T
$$

must be conserved over time. That is, they must satisfy the model property $\dot{N}(t)=0$. This yields the extra conditions

$$
\begin{aligned}
& u_{T}\left(T_{\min }, t\right)=-v_{T}\left(T_{\min }, t\right) \\
& v_{T}\left(T_{\max }, t\right)=-u_{T}\left(T_{\max }, t\right)
\end{aligned}
$$

Figure 3 illustrates the improved modeling capabilities of adding the diffusive term. 


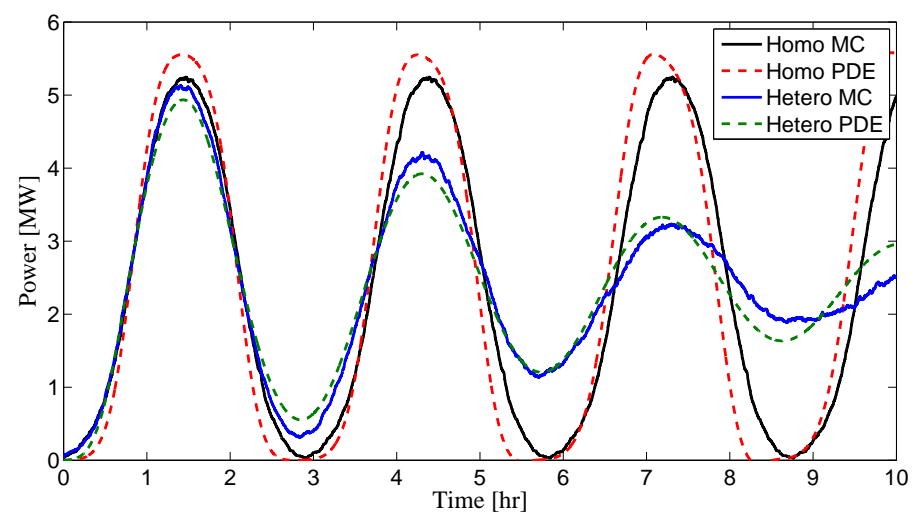

Figure 3. Comparison of aggregate TCL power for the homogeneous and heterogeneous populations, using the 1,000 individual TCLs in the Monte Carlo (MC) model, and the PDE models. The heterogeneous PDE model captures the damped oscillations exhibited by the 1,000 individual TCL models.

However, while it was in theory possible to compute the parameters in the heterogeneous model if one had access to knowledge of $\bar{C}, \bar{R}$ and $\bar{P}$, it is not possible to compute the parameters in (20)-(21) in a similar manner. Therefore, we instead develop a system identification scheme to determine the parameters based on online system measurements.

\section{PARAMETER IDENTIFICATION}

Consider the aggregated power consumption of the TCL population given by (19). Differentiating (19) with respect to

Table 1. Model parameter values

\begin{tabular}{lll}
\hline \hline Parameter & Value & Description \\
\hline $\bar{R}$ & $2{ }^{\circ} \mathrm{C} / \mathrm{kW}$ & Mean thermal resistance \\
$\bar{C}$ & $10 \mathrm{kWh} /{ }^{\circ} \mathrm{C}$ & Mean thermal capacitance \\
$\bar{P}$ & $14 \mathrm{~kW}$ & Mean thermal power \\
$T_{\infty}$ & $32{ }^{\circ} \mathrm{C}$ & Mean ambient temperature \\
$T_{s p}$ & $20{ }^{\circ} \mathrm{C}$ & Temperature setpoint \\
$\Theta$ & $1{ }^{\circ} \mathrm{C}$ & Temperature deadband width \\
$\eta$ & 2.5 & Coefficient of performance \\
$\beta$ & 0.0145 & Diffusivity \\
$u_{0}(T)$ & 0 & Initial distribution of on TCLs \\
$v_{0}(T)$ & $\mathcal{N}\left(20^{\circ} \mathrm{C},\left(0.2^{\circ} \mathrm{C}\right)^{2}\right)$ & Initial distribution of off TCLs \\
\hline \hline
\end{tabular}

time, followed by integration by parts yields

$$
\begin{aligned}
\frac{\eta}{\bar{P}} \dot{P}(t)= & \frac{\partial}{\partial t} \int_{T_{\min }}^{T_{\max }} u(T, t) d T \\
= & \int_{T_{\min }}^{T_{\max }}\left[\alpha \lambda(T) u_{T}(T, t)+\alpha u(T, t)+\beta u_{T T}(T, t)\right] d T \\
= & \alpha\left[\left.\lambda(T) u(T, t)\right|_{T_{\min }} ^{T_{\max }}-\int_{T_{\min }}^{T_{\max }}\left(\lambda_{T}(T)-1\right) u(T, t) d T\right] \\
& +\left.\beta u_{T}(T, t)\right|_{T_{\min }} ^{T_{\max }}
\end{aligned}
$$

Inserting $\lambda(T)=T+\bar{R} \bar{P}-T_{\infty}$ and $\lambda_{T}(T)=1$ into (27) and rearranging, we get

$$
\begin{aligned}
\frac{\eta}{\bar{P}} \dot{P}(t)= & \alpha\left[T_{\max } u\left(T_{\max }, t\right)-T_{\min } u\left(T_{\min }, t\right)\right] \\
& +\alpha b\left[u\left(T_{\max }, t\right)-u\left(T_{\min }, t\right)\right] \\
& +\beta\left[u_{T}\left(T_{\max }, t\right)-u_{T}\left(T_{\min }, t\right)\right] \\
= & \alpha \phi_{1}(t)+\alpha b \phi_{2}(t)+\beta \phi_{3}(t)
\end{aligned}
$$

where $b=\bar{R} \bar{P}-T_{\infty}$ and $\phi_{1}, \phi_{2}, \phi_{3}$ are given by the respective bracketed terms. Equation (29) provides a parametric model that is linear in the uncertain parameters $\alpha, \alpha \cdot b$, and $\beta$.

To utilize this parametric model, we require measurements $u\left(T_{\min }, t\right), u\left(T_{\max }, t\right), u_{T}\left(T_{\min }, t\right), u_{T}\left(T_{\max }, t\right)$. In the TCL application, this means TCLs announce themselves when they turn on and off, thus providing measurements of $u\left(T_{\min }, t\right)$ and $u\left(T_{\max }, t\right)$. For $u_{T}\left(T_{\min }, t\right)$ and $u_{T}\left(T_{\max }, t\right)$, we use finite differences. That is, TCLs must also announce themselves when their temperature is slightly below (and slightly above) the upper (and lower) limit of their deadband, when cooling (and when heating).

Since the parametric model contains time derivatives of measured signals, we employ the standard technique of filtering to avoid direct differentiation (see, e.g. Sec. 2.4.1 of [16]):

$$
\begin{aligned}
& \dot{\chi}_{1}=-a \chi_{1}+\frac{\eta}{\bar{P}} P(t) \\
& \dot{\chi}_{2}=-a \chi_{2}+\phi_{1}(t) \\
& \dot{\chi}_{3}=-a \chi_{3}+\phi_{2}(t) \\
& \dot{\chi}_{4}=-a \chi_{4}+\phi_{3}(t)
\end{aligned}
$$

where $a>0$ is a constant chosen by the user. This constant is chosen large enough to satisfy a standard persistency of excitation condition, yet small enough to attenuate measurement noise. The filtered version of (29) becomes

$$
-a \chi_{1}+P(t)=\alpha \chi_{2}+\alpha b \chi_{3}+\beta \chi_{4}
$$

We choose the parameter vector

$$
\hat{\theta}=[\hat{\alpha} \widehat{\alpha b} \hat{\beta}]^{T}
$$


Note that $(\hat{\alpha}, \hat{b}, \hat{\beta})$ can be uniquely determined from $(\hat{\alpha}, \widehat{\alpha b}, \hat{\beta})$. We define the regressor as

$$
\phi=\left[\begin{array}{lll}
\chi_{2} & \chi_{3} & \chi_{4}
\end{array}\right]^{T}
$$

These definitions permit us to rewrite the parametric model (34) into the form

$$
-a \chi_{1}+P(t)=\hat{\theta}^{T} \phi(t)
$$

and deploy the following standard least squares estimator with normalization [16]:

$$
\begin{aligned}
\varepsilon & =-a \chi+P(t)-\hat{\theta}^{T} \phi \\
\dot{\hat{\theta}} & =\frac{1}{1+\gamma \phi^{T} \phi} \Gamma \phi \varepsilon \\
\dot{\Gamma} & =-\frac{1}{1+\gamma \phi^{T} \phi} \Gamma \phi \phi^{T} \Gamma, \quad \Gamma(0)=\Gamma_{0}=\Gamma_{0}^{T}
\end{aligned}
$$

where $\gamma>0$ and $\Gamma_{0}>0$ are user-determined constants.

\section{SIMULATION RESULTS}

This section illustrates the parameter identification scheme proposed above. First, we estimate the true parameters in a model of the form (20)-(26) using only measurements of the aggregate power and the boundary values (22)-(26). The true parameters were set to $\alpha=.0496, b=15.5$ and $\beta=.0145$. The result of executing (38)-(40) with $\Gamma_{0}=0.1 I$ and $a=0.3$ is shown in Fig. 4 and 5. As can be seen, the two first parameter estimates converge to the true values, whereas $\hat{\beta}$ converges to a slightly larger value than 0.0145 . This is likely due to using first-order finite-differences in the computation of $u_{T}\left(T_{\max }\right)$ and $u_{T}\left(T_{\min }\right)$, which degrade the second-order accuracy of the Crank-Nicolson scheme used to solve the PDE model.

Next, the same simulation is attempted with noisy measurements. Gaussian white noise with a standard deviation of 1 percent of the maximum amplitude of $u(T, t)$ was added to $u(T, t)$ at all points in a grid with density $\delta T=.02^{\circ} \mathrm{C}, \delta t=10^{-3} \mathrm{~h}$. All other parameters were identical to the previous simulation. The parameter convergence is shown in Figure 6. As can be seen, the parameters still converge to the true values, although some high-frequency oscillations occur in the estimation of $\hat{\alpha}$ around 1 hour into the simulation. The estimation of $\dot{P}(t)$ is practically indistinguishable from the previous case in Fig. 5.

Finally, we apply the identification scheme to a Monte Carlo-simulation model of a large population of TCLs. We simulate 1000 TCLs with the parameter values given in Table 1, except that $C_{i}$ are drawn from a normal distribution with mean 10 $\mathrm{kWh} /{ }^{\circ} \mathrm{C}$ and standard deviation $1 \mathrm{kWh} /{ }^{\circ} \mathrm{C}$. The aggregate power is measured according to (3). To obtain $u\left(T_{\min }, t\right)$ and $u\left(T_{\max }, t\right)$,

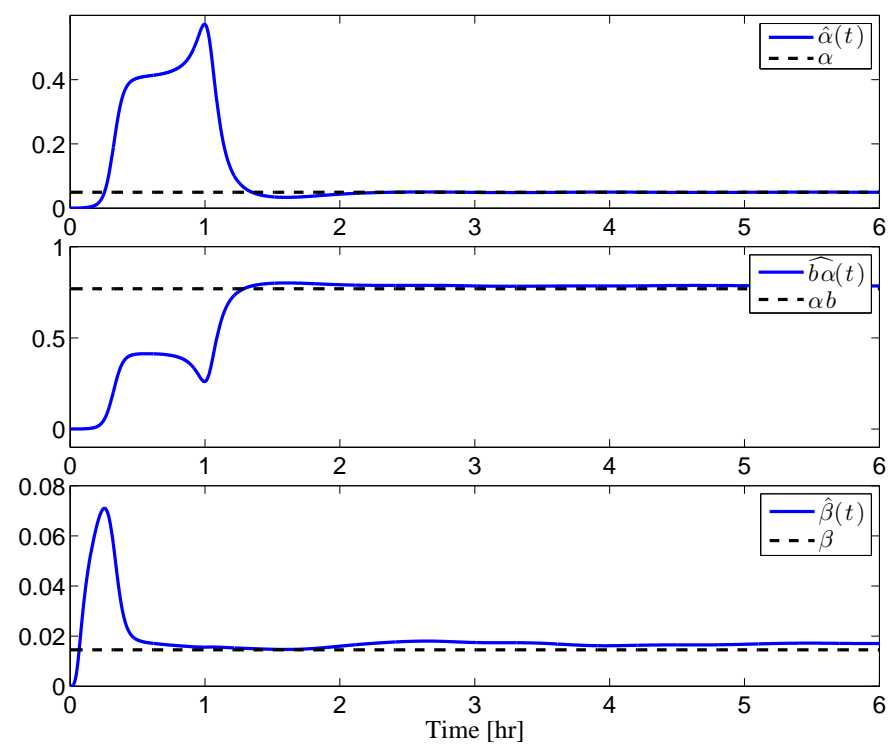

Figure 4. Parameter estimation, noise-free case.

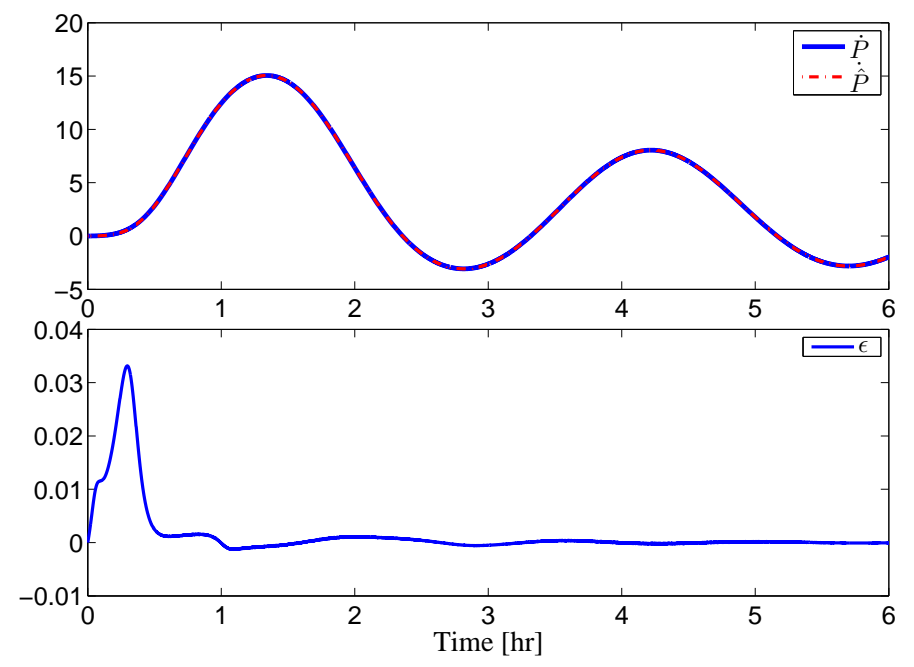

Figure 5. Estimation of $\dot{P}(t)$ generated from a simulation of (20)-(26); top: estimate and true signal; bottom: estimation error $\varepsilon$.

we measure the number of TCLs which switch on and off, respectively. To obtain $u_{T}\left(T_{\min }, t\right)$ and $u_{T}\left(T_{\max }, t\right)$, we measure the number of TCLs with temperature $T_{i} \in\left[T_{\min }, T_{\min }+\delta T\right]$ and $T_{i} \in\left[T_{\max }-\delta T, T_{\max }\right]$ and use first-order finite differences. The result of estimating the time derivative of the aggregated power and the corresponding parameter estimates are shown in Fig. 7 and 8 , respectively.

The figures indicate that the parameter estimates are noisy, although the model estimates $\dot{P}$ quite well. This may be attributed to (i) the measurements themselves being very noisy, indicating the need for effective filtering, and (ii) approximation errors incurred by utilizing an aggregated PDE model. Neverthe- 

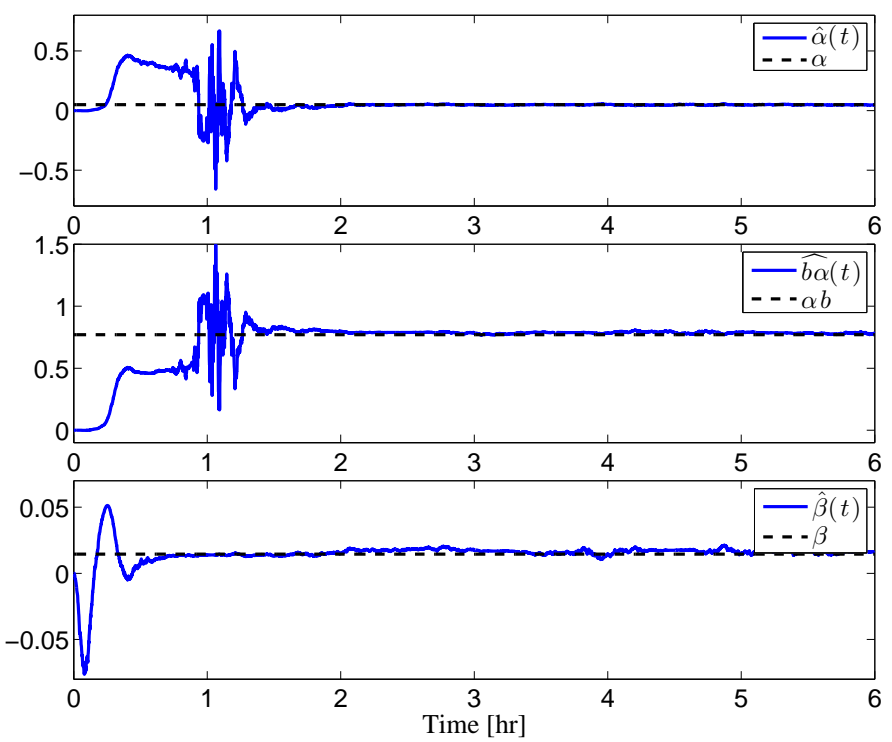

Figure 6. Parameter estimation, noisy PDE simulation.

less, the estimates are bounded and converge within some neighborhood of the true values, whose size can be adjusted by tuning $a$ in (30)-(33), $\gamma$ in (40), and $\Gamma_{0}$ in (40).

\section{CONCLUSION}

This paper presents PDE-based models and a parameter identification algorithm for aggregated heterogeneous populations of thermostatically controlled loads. First, a two-state boundary-coupled hyperbolic PDE model for homogenous TCL populations was derived, which was then extended to heterogeneous populations by including a diffusive term. Second, a novel parameter identification scheme was derived for the PDE model structure, utilizing only boundary measurements and aggregated power measurements. Simulation results highlight the various properties of the models and identification algorithm, including application of the algorithm on data generated by Monte Carlo simulation. From the experiments it is not easy to say anything definite regarding the rate of convergence, but persistent excitation does seem to be quite important for identification of the diffusion coefficient.

This work may be extended in several interesting and important ways. First, one may seek to derive other parameter identification schemes using different measurement sets, e.g. aggregate power only or full-state measurements. Second, estimation of the PDE states $u$ and $v$ (i.e., state observer designs) are desirable for monitoring the TCL population with a minimal sensing infrastructure. Third, ultimately feedback control schemes must be developed to shape aggregate power demand, using the aforementioned state and parameter estimates. Fourth, there is currently a dearth of published results exemplifying such modelbased estimation and control algorithms on real-world data. Fi-
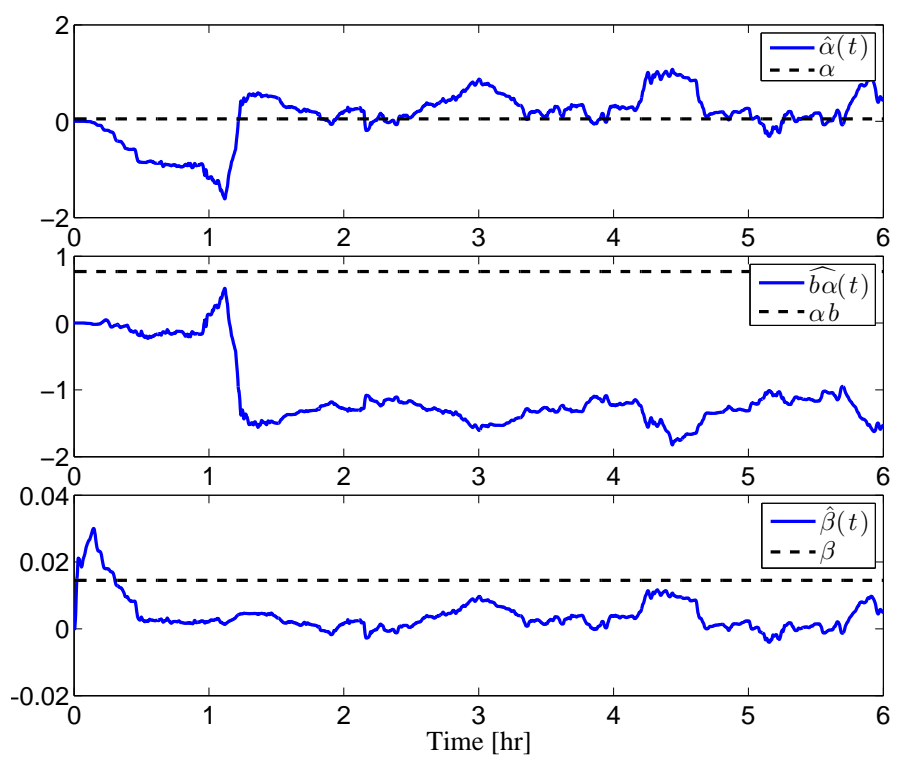

Figure 7. Parameter estimation based on data from a population of TCLs.

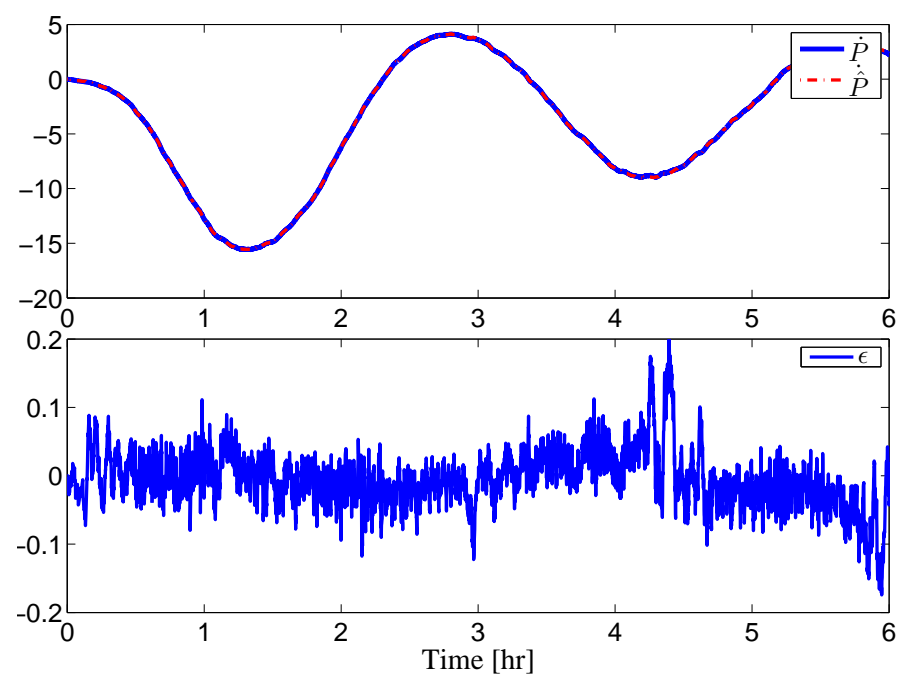

Figure 8. Estimation of $\dot{P}(t)$ generated from a simulation of 1000 TCLs; top: estimate and true signal; bottom: estimation error $\varepsilon$.

nally, the fundamental PDE-based modeling abstraction can be extended to other flexible loads, such as charging/discharging of aggregated plug-in electric vehicles $[17,18]$.

\section{REFERENCES}

[1] Mohsenian-Rad, A., v.W.S. Wong, Jatskevich, J., Schober, R., and Leon-Garcia, A., 2010. "Autonomous demand-side management based on game-theoretic energy consumption scheduling for the future smart grid". IEEE Transactions on Smart Grid, 1(3), pp. 320-331. 
[2] Strbac, G., 2008. "Demand side management: Benefits and challenges". Energy Policy, 36(12), pp. 4419-4426.

[3] Short, J., Infield, D. G., and Freris, L. L., 2007. "Stabilization of grid frequency through dynamic demand control". IEEE Transactions on Power Systems, 22(3), pp. 12841293.

[4] Malhame, R., and Chong, C., 1985. "Electric load model synthesis by diffusion approximation of a higher-order hybrid-state stochastic system". IEEE Transactions on Automatic Control, 30(9), pp. 854-860.

[5] Callaway, D., 2009. "Tapping the energy storage potential in electric loads to deliver load following and regulation, with application to wind energy". Energy Conversion and Management, 50(9), pp. 1389-1400.

[6] Callaway, D., and Hiskens, I., 2011. "Achieving controllability of electric loads". Proceedings of the IEEE, 99(1), pp. 184-199.

[7] Kundu, S., Sinitsyn, N., Backhaus, S., and Hiskens, I. A., 2011. "Modeling and control of thermostaticallycontrolled-loads". In Proc. of the 17th Power Systems Computations Conference.

[8] Kundu, S., and Sinitsyn, N., 2012. "Safe protocol for controlling power consumption by a heterogeneous population of loads". In Proc. of 2012 American Control Conference.

[9] Perfumo, C., Kofman, E., Braslavsky, J., and Ward, J., 2012. "Load management: Model-based control of aggregate power for populations of thermostatically controlled loads". Energy Conversion and Management, 55(1), pp. 36-48.

[10] Bashash, S., and Fathy, H., 2012. "Modeling and control of aggregate air conditioning loads for robust renewable power management". IEEE Transactions on Control Systems Technology, PP(99), pp. 1-10.

[11] Zhang, W., Lian, J., Chang, C.-Y., Kalsi, K., and Sun, Y., 2012. "Reduced-order modeling of aggregated thermostatic loads with demand response". In Proc. of 51st IEEE Conference on Decision and Control.

[12] McDonald, H., 2008. "Adaptive intelligent power systems: Active distribution networks". Energy Policy(36), pp. 4346-4351.

[13] Andersen, P., Pedersen, T. S., and Nielsen, K. M., 2012. "Observer based model identification of heat pumps in a smart grid". In Proc. of Multiconf. Systems and Control.

[14] Lu, N., Chassin, D. P., and Widergren, S. E., 2005. "Modeling uncertainties in aggregated thermostatically controlled loads using a state queueing model". IEEE Transactions on Power Systems, 20(2), pp. 725-733.

[15] Mathieu, J. L., Koch, S., and Callaway, D. S., 2013. "State estimation and control of electric loads to manage real-time energy imbalance". IEEE Transactions on Power Systems, 28(1), Feb., pp. $430-440$.

[16] Ioannou, P., and Sun, J., 1996. Robust Adaptive Control. Prentice-Hall.

[17] Bashash, S., Moura, S. J., Forman, J. C., and Fathy, H. K., 2011. "Plug-in hybrid electric vehicle charge pattern optimization for energy cost and battery longevity". Journal of Power Sources, 196(1), pp. 541 - 549.

[18] Bashash, S., Moura, S. J., and Fathy, H. K., 2011. "On the aggregate grid load imposed by battery health-conscious charging of plug-in hybrid electric vehicles". Journal of Power Sources, 196(20), pp. 8747 - 8754. 